\title{
Peculiarities of Homeostatic and Genetic Profile in Women With Miscarriage Contaminated With Hydroxybenzol
}

\author{
Kazakova O.A. ${ }^{1, *}$ Alekseev V.B. ${ }^{1}$ Sinitsyna O.O. ${ }^{2}$ Dolgikh O.V. ${ }^{1}$ \\ ${ }^{1}$ Federal Scientific Research Center for Medical and Preventive Health Risk Management Technologies, Perm, Russia \\ ${ }^{2}$ Federal Scientific Center of Hygiene named after F.F. Erisman, Moscow, Russia
}

*Corresponding author. Email: chakina2011@yandex.ru

\begin{abstract}
The analysis of the features of homeostatic markers and genetic polymorphism in women of fertile age, whose anamnesis is characterized by the presence of reproductive disorders in the form of miscarriage associated with the excessive contamination of biomedia with hydroxybenzene of technogenic origin. Changes in the parameters of regulation and metabolism were revealed in the form of an increase in the level of phosphorus, AST to the upper limit of the norm, and a decrease in the level of total cholesterol. It was found that the $\mathrm{C}$ allele and the $\mathrm{C} / \mathrm{C}$ genotype of peroxisome gene PPARG Pro12Ala rs 1801282 act as factors of undesirable reproductive events (miscarriage). At the same time, the G/G genotype of the SULT1A1 gene Arg213His rs9282861 acts as a factor associated with the conditions of increased blood contamination with phenol, abolishing the synthesis of sex steroid, modifying lipoprotein metabolism with a drop in total cholesterol in women with miscarriage.
\end{abstract}

Keywords: miscarriage, hydroxybenzene contamination, homeostasis markers, SULT1A1 gene, PPARG gene

Peroxisome proliferation activating receptors

\section{INTRODUCTION}

The study of the miscarriage problem is of particular relevance, taking into account the modern challenges to the health of women who are daily exposed to the adverse conditions of external environment of anthropogenic origin [1].

The negative impact of hydroxybenzene, which enters the woman's body by aerogenic means can also be attributed to the number of unfavorable technogenic factors. Hydroxybenzene is a hormone-like toxicant that disrupts the level of natural steroid hormones triggering a cascade of reactions in the body, affecting the immune, endocrine, and hormonal systems [2-4].

During pregnancy, the female body undergoes significant changes, which are manifested not only in changes in the immune and hormonal indicators, but also in homeostasis indicators (biochemical indicators). Changes in the level of expression of biochemical parameters may indicate ongoing disturbances in the body system balance [5].

The genetic markers act as predisposing factors for the development of various pathologies manifested under the influence of unfavorable environmental and lifestyle factors.
(PPARs) are a group of nuclear receptors that function as a transcription factor. PPARs play an essential role in the regulation of cell differentiation, glucose and lipid metabolism, have an anti-inflammatory effect, an effect on the ovaries and placenta, which can serve as a link between carbohydrate and lipid metabolism, inflammatory response, and reproductive function. Divakova and Ruzhilo suggest that the defects in regulatory genes such as PPARs are the cause of a cascade of metabolic and hormonal disorders [6].

Sulfotransferases (SULTs) form a superfamily of genes that are involved in Phase II biotransformation of endobiotics and xenobiotics through sulfonation. Sulfoconjugation transforms such compounds into a more hydrophilic form, which facilitates their elimination.

By influencing detoxification mechanisms, SULTs are involved in the initiation of hepatotoxicity when any highly reactive electrophiles produced by sulfonation are not restored [7]. SULT1A1 is capable of sulfonating a wide variety of phenolic compounds (hydroxybenzenes) such as p-nitrophenol, as well as endogenous compounds such as estrogen, thyroid hormones, and neurotransmitters [8]. The purpose of the work is to assess the features of biochemical and genetic profile of women with recurrent miscarriage exposed to hydroxybenzene. 


\section{METHODS AND MATERIALS}

The study involved 200 women of reproductive age permanently residing in the territory with the increased aerogenic pollution with hydroxybenzene more than $>1.0$ threshold limit value - time weighted average. There were identified 4 samples: Observation 1 60 women with reproductive disorders at the age of $31.19 \pm 0.89$ years old contaminated with hydroxybenzene in the blood above the reference level (> $0.016 \mathrm{mg} / \mathrm{cm}^{3}$ ); Observation $2-60$ women with reproductive disorders at the age of $31.25 \pm 0.77$ years old, not contaminated with hydroxybenzene; Control 1 - 31 conditionally healthy women aged $33.17 \pm 1.06$, contaminated with hydroxybenzene above the reference level (> $0.016 \mathrm{mg} / \mathrm{cm}^{3}$ ); Control 2 - 29 apparently healthy women aged $34.10 \pm 0.92$, without blood contamination with hydroxybenzene.

The level of contamination of biological media (blood) with hydroxybenzene was determined by gas chromatography with the inductively coupled plasma on the Kristall 5000 chromatograph (Russia). The levels of biochemical parameters (phosphorus, iron, ACAT) were determined photometrically using Keylab (BPS Biosed, Italy) and Humalyzer 2000 (Human, Germany) devices. The polymorphism of the SULT1A1 sulftransferase genes Arg213His rs9282861 and the peroxisome receptor gene PPARG Pro12Ala rs1801282 was evaluated using BioRAD CFX96 device in real time with the allelic discrimination in the TaqMAN software.

Table 1 Features of levels of biochemical indicators in the study groups
The statistical data processing was carried out in the Statistica10.0 program. The results are presented as $\mathrm{X}$ mean, SE-error of the mean $(\mathrm{X} \pm \mathrm{SE})$. The distribution normality was estimated based on the $\mathrm{W}$ of ShapiroWilk test. The level of significance was established using the correction for multiple comparisons Bonferoni, which makes it possible to exclude type I error, i.e. "false alarm", while the differences $\mathrm{p}<0.0083$ were considered significant. As for the samples with a normal distribution, the results of comparison by the Student's t test were considered significant, for samples with an abnormal distribution by the U-Mann-Whitney test. The genetic data were analyzed using Multiplicative and General Inheritance Models, with the identification of compliance with the equilibrium of the Hardy-Weindberg law. A cross-classification analysis of the "sensitivity marker - exposure marker - effect marker" models was carried out with the help of online SNPstats of the Catalan Cancer Institute.

\section{RESULTS}

According to the results of the assessment of homeostatic parameters presented in TABLE 1 , it was found out that all the studied biochemical parameters in all studied groups, with the exception of Aspartate Aminotransferase (ASAT) in the "Observation 1" group (women with reproductive system pathologies with the increased blood contamination with phenol), which did not differ significantly from the upper limit, varied within the normal range.

\begin{tabular}{|l|c|c|c|c|c|}
\hline \multicolumn{1}{|c|}{ Indicator } & Norm & Observation 1 & Observation 2 & Control 1 & Control 2 \\
\hline Phosphorus & $0.8-1.62 \mathrm{mmol} / \mathrm{dm}^{3}$ & $1.41 \pm 0.03$ & $1.42 \pm 0.09^{*}$ & $1.13 \pm 0.03$ & $1.17 \pm 0.04$ \\
\hline Iron & $6.6-26.0 \mathrm{mmol} / \mathrm{dm}^{3}$ & $13.33 \pm 0.96$ & $12.61 \pm 1.69$ & $14.21 \pm 1.18^{*}$ & $14.57 \pm 1.19^{*}$ \\
\hline ALT & $0-32 \mathrm{E} / \mathrm{dm}^{3}$ & $26.78 \pm 7.67 *$ & $20.86 \pm 3.55^{*}$ & $13.83 \pm 1.33^{*}$ & $14.46 \pm 1.20^{*}$ \\
\hline ASA & $0-31 \mathrm{E} / \mathrm{dm}^{3}$ & $31.53 \pm 5.22^{*} \uparrow$ & $22.93 \pm 1.38$ & $17.52 \pm 1.02$ & $22.29 \pm 1.37 *$ \\
\hline$\gamma$ - GT & $7-32 \mathrm{E} / \mathrm{dm}^{3}$ & $24.78 \pm 3.79^{*}$ & $22.00 \pm 2.08$ & $21.55 \pm 2.11^{*}$ & $20.43 \pm 1.79 *$ \\
\hline Total cholesterol & $0-5.16 \mathrm{mmol} / \mathrm{dm}^{3}$ & $4.21 \pm 0.13$ & $4.63 \pm 0.32$ & $4.69 \pm 0.16$ & $5.12 \pm 0.26^{*}$ \\
\hline
\end{tabular}

Note: $*$ - the indicator corresponds to the normal Shapiro-Wilk distribution, $\uparrow$ - the indicator exceeds the upper limit of the norm, $\downarrow$ - the indicator is less than the lower limit of the norm, $* *$ - the differences with the norm are significant.

Pairwise comparisons revealed differences between women with reproductive disorders and increased contamination of biological media (blood) with phenol, and women with similar disorders, but the level of phenol was within the physiological limit (Observation 1 - Observation 2): Despite a slight difference of 1.05 
times in the level of calcium in the blood these differences were significant according to the U-test and corresponded to the established level of significance, taking into account the correction ( $\mathrm{p}<0.0083)$.

The comparison of the groups of apparently healthy women with increased contamination of biological media with phenol above the permissible level and conditionally healthy women with the level of phenol within the physiological limit (Control 1 - Control 2) established significant differences in ASA levels by 1.27 times according to the $\mathrm{U}-$ criterion.

The comparison of women with reproductive disorders and conditionally healthy women with increased contamination of biological media biological media with phenol (Observation 1-Control 1) revealed a significant difference in the level of calcium by 1.08 times, by the level of phosphorus by 1.25 times, by the level of ALT by 1.93 times, according to the ASA level by 1.80 times according to the U-criterion.

The comparison of women with reproductive disorders and high level of phenol in the blood with conditionally healthy non-contaminated with phenol (Observation 1 Control 2) revealed significant differences in the level of phosphorus by 1.21 times, ASA by 1.42 times, and total cholesterol by 1.23 times (U-test).

Pairwise comparisons of groups of women with reproductive disorders and an acceptable level of phenol and conventionally healthy ones contaminated

Table 2 Frequencies of genotypes and alleles of studied regulation genes pparg in the study groups and their compliance with the hardy-weinberg balance law with phenol (Observation 2-Control1) revealed significant differences in the levels of phosphorus (1.26 times) and ACAT (1.31 times) according to the U-test.

The study of the regulation gene polymorphism and comparison of genotype frequencies between the groups of women with reproductive disorders and conditionally healthy, whose blood phenol was within the physiological level (Observation 1-Control 1),made it possible to reveal significant differences in polymorphism of the peroxisome receptor gene PPARG Pro12Ala 1801282 associated with a decrease in the frequency of the $\mathrm{G}$ allele by 3.0 times, the prevalence of the homozygous $\mathrm{C} / \mathrm{C}$ genotype by 2.31 times, the decrease in the frequency of the heterozygous $\mathrm{C} / \mathrm{G}$ genotype by 2.67 times, as well as the homozygous $\mathrm{G} / \mathrm{G}$ genotype by 5.33 times (Table 2)

According to the established level of significance, it was shown that an excessive level of blood contamination with phenol increases the likelihood of developing adverse reproductive events (Observation1Control1) associated with the PPARG Pro12Ala $1801282 \mathrm{C}$ allele polymorphism $(\mathrm{OR}=4.45$; $\mathrm{CI}$ : 1.44 13.47) and $\mathrm{C} / \mathrm{C}$ genotype $(\mathrm{OR}=6.73$; $\mathrm{CI}: 1.44-31.40)$ (Table 2). For the SULT1A1 Arg213His 9282861 gene, no significant differences in allele and genotype frequencies were found between the studied groups (Table 3).

\begin{tabular}{|c|c|c|c|c|c|c|c|c|c|c|c|c|}
\hline Groups & Obs & rvati & 1 & & & & Obs & rvat & 2 & & & \\
\hline \multirow[b]{2}{*}{ Gene / polymorphism / rs. } & \multicolumn{3}{|c|}{ Genotypes } & \multicolumn{2}{|c|}{ Alleles } & \multirow[b]{2}{*}{$\mathrm{p}$} & \multicolumn{3}{|c|}{ Genotypes } & \multicolumn{2}{|c|}{ Alleles } & \multirow[b]{2}{*}{$\mathrm{p}$} \\
\hline & G1 & Get & $\mathrm{G} 2$ & A1 & $\mathrm{A} 2$ & & G1 & Get & $\mathrm{G} 2$ & A1 & $\mathrm{A} 2$ & \\
\hline PPARG Pro12Ala 1801282 & 77 & 21 & 2 & 88 & 13 & + & 83 & 17 & 0 & 92 & 8 & + \\
\hline Groups & \multicolumn{6}{|c|}{ Control 1} & \multicolumn{6}{|c|}{ Control 2} \\
\hline \multirow[b]{2}{*}{ Gene / polymorphism / rs. } & \multicolumn{3}{|c|}{ Genotypes } & \multicolumn{2}{|c|}{ Alleles } & & \multicolumn{3}{|c|}{ Genotypes } & \multicolumn{2}{|c|}{ Alleles } & \\
\hline & G1 & Get & $\mathrm{G} 2$ & A1 & A2 & $\mathrm{p}$ & G1 & Get & $\mathrm{G} 2$ & A1 & $\mathrm{A} 2$ & $\mathrm{p}$ \\
\hline PPARG Pro12Ala 1801282 & 33 & 56 & 11 & 61 & 39 & + & 60 & 40 & 0 & 80 & 20 & + \\
\hline
\end{tabular}

Note: G1 - normal homozygote, Get - heterozygote, G2 - mutant homozygote, A1 - normal allele, A2 - mutant allele, p - significance level for HWE, "+" - the HWE law is not violated, "-" - the HWE law is violated. 
Table 3 Frequencies of genotypes and alleles of the gene for detoxification of phenolic compounds sult1a1 in the studied groups and their compliance with the law of equilibrium of hardy-weinberg

\begin{tabular}{|c|c|c|c|c|c|c|c|c|c|c|c|c|}
\hline \multirow[b]{3}{*}{ Gene / polymorphism / rs. } & \multicolumn{6}{|c|}{ Observation 1} & \multicolumn{6}{|c|}{ Observation 2} \\
\hline & \multicolumn{3}{|c|}{ Genotypes } & \multicolumn{2}{|c|}{ Alleles } & \multirow[b]{2}{*}{$\mathrm{p}$} & \multicolumn{3}{|c|}{ Genotypes } & \multicolumn{2}{|c|}{ Alleles } & \multirow[b]{2}{*}{$\mathrm{p}$} \\
\hline & G1 & Get & G2 & A1 & A2 & & G1 & Get & G2 & A1 & A2 & \\
\hline SULT1A1 Arg213His 9282861 & 27 & 53 & 20 & 53 & 47 & + & 18 & 58 & 23 & 48 & 53 & + \\
\hline Groups & \multicolumn{6}{|c|}{ Control 1} & \multicolumn{6}{|c|}{ Control 2} \\
\hline & \multicolumn{3}{|c|}{ Genotypes } & \multicolumn{2}{|c|}{ Alleles } & & \multicolumn{3}{|c|}{ Genotypes } & \multicolumn{2}{|c|}{ Alleles } & \\
\hline Gene / polymorphism / rs. & G1 & Get & G2 & A1 & $\mathrm{A} 2$ & $\mathrm{p}$ & G1 & Get & G2 & $\mathrm{A} 1$ & $\mathrm{~A} 2$ & $\mathrm{p}$ \\
\hline SULT1A1 Arg213His 9282861 & 51 & 26 & 23 & 64 & 36 & - & 27 & 41 & 32 & 48 & 52 & + \\
\hline
\end{tabular}

Note: G1 - normal homozygote, Get - heterozygote, G2 - mutant homozygote, A1 - normal allele, A2 - mutant allele, p - significance level for HWE, "+" - the HWE law is not violated, "-" - the HWE law is violated.

The dependence of the total cholesterol level on the genotypes of the SULT1A1 Arg213His rs9282861 sulftransferase gene under conditions of various blood contamination with phenol - sensitivity marker (gene) exposure marker (phenol in the blood) - response marker (total cholesterol). For example, under

Table 4. Dependence parameters in the system "sensitivity marker - exposition marker - response marker"

\begin{tabular}{|l|c|c|c|c|}
\hline \multicolumn{2}{|c|}{ Gene is the answer } & \multicolumn{2}{c|}{ Phenol above normal limit (>0.016) } & \multicolumn{2}{l|}{ Phenol within normal limits (0-0.016) } \\
\hline SULT1A1 - total cholesterol & X \pm SE & Differences (CI 95 \%) & X \pm SE & Differences (CI 95 \%) \\
\hline G/G & $86.91 \pm 12.64$ & 0.00 & $221.91 \pm 43.98$ & $135.00(57.61-212.40)$ \\
\hline G/A & $95.53 \pm 17.95$ & $8.61(-37.86-55.09)$ & $82.55 \pm 24.82$ & $-4.36(-53.46-44.73)$ \\
\hline A/A & $155.08 \pm 26.46$ & $68.17(15.63-120.71)$ & $91.27 \pm 23.18$ & $4.35(-60.17-68.88)$ \\
\hline Interaction $\mathrm{p}=0,019$ & & & & \\
\hline
\end{tabular}

Note: $\mathrm{X}$ is a mean, $\mathrm{SE}$ is an error of the mean, $\mathrm{CI}$ is a confidence interval, $\mathrm{p}$ is a significance level.

\section{DISCUSSION}

Phosphorus homeostasis is less susceptible to change than calcium, but it is dependent on it, therefore it can conditions of increased blood contamination with phenol, the homozygous $\mathrm{G} / \mathrm{G}$ genotype acted as a factor canceling the synthesis of sex steroids, modifying lipoprotein metabolism with a drop in total cholesterol in women with miscarriage (Table 4). also be associated with the development of miscarriage [10] and its serum insufficiency can be associated with premature uterine contractility [11]

Elevated levels of ALT and ASA, which go beyond the upper limit of the norm in women with reproductive 
disorders and increased blood contamination with phenol, may testify in favor of hepatotoxicity of phenols, since the liver is the primary organ where the processes of detoxification of xenobiotics occur and alanine aminotransferase and aspartic aminotransferase are associated with the processes in the liver [12].

Changes in total cholesterol levels are associated with reproductive disorders [13]. Cholesterol is the source for production of all steroid hormones. Due to the fact that phenols are substances mimicking sex hormones that replace natural estrogens, the homeostatic need for cholesterol decreases forming a deficiency in total cholesterol the synthesis of which is canceled by phenols in women with reproductive disorders.

\section{CONCLUSION}

The formation of pathology of the reproductive system in women of fertile age in conditions of excessive blood contamination with phenol is accompanied by a decrease in the level of total cholesterol in the blood. An inverse reliable dependence of the expression of total cholesterol on the level of phenol in the blood was established. Peroxisome receptor gene PPARG Pro12Ala 1801282, its C allele (OR $=4.45$; CI: $1.44-$ 13.47) and $\mathrm{C} / \mathrm{C}$ genotype (OR $=6.73$; $\mathrm{CI}: 1.44-31.40)$ are decisive factors in the development of reproductive disorders, aggravated by increased blood contamination with phenol.

The SULT1A1 Arg213His rs9282861 sulftransferase gene and its homozygous $\mathrm{G} / \mathrm{G}$ genotype act as a factor that cancels the synthesis of sex steroids modifying lipoprotein metabolism with a drop in total cholesterol in women with miscarriage.I $t$ is recommended to use the studied markers of homeostasis (total cholesterol, ALAT, ACAT) and genes for regulation (PPARG Pro12Ala rs1801282) and detoxification of phenolic compounds (SULT1A1 Arg213His rs9282861) to screen for the development of miscarriage in conditions of excessive contamination with hydroxybenzenes.

\section{REFERENCES}

[1] L.A. Balabanova, Environmental and professional factors in the Genesis of reproductive disorders, Pract. Med. 30 (2008) 16.

[2] O.A. Kazakova, O.V. Dolgikh, O.O. Sinitsina, Immune and genetic status of women with reproductive disorders in the case of contamination of the biological medium with phenol, Hygiene and sanitat. 99(1) (2020) 90-96.

[3] V.O. Chernov, N.V. Artymuk, L.N. Danilova, Hormone-like xenobiotics and gynecological problems. Literature review, Mother and child in Kuzbass 2(73) (2018) 20-26.

[4] A.I. Nikitin, Hormone-like xenobiotics and their role in human reproductive pathology, Human ecol. 2 (2006) 17-23.

[5] S.G. Dzugkoev, A.I. Tedtoeva, F.S. Dzugkoeva et al., Evaluation of biochemical parameters in pregnant women with metabolic disorders: results of a comparative study, Obstetr. Gynecol. Reproduct. 11(2) (2017) 12-17.

[6] O.S. Ruzhilo, T.S. Divakova, Influence of polymorphic variants of receptor genes that activate peroxisome proliferation of PPARa and PAPRGC1a on the development of polycystic ovary syndrome, Vest. Vmgub. 12(3) (2013) 78-83.

[7] R.M. Weinshilboum et al., Sulfation and sulfotransferases 1: sulfotransferase molecular biology: cDNAs and genes, FASEB J. 11(1) (1997) 3-14.

[8] N. Gamage et al., Human sulfotransferases and their role in chemical metabolism, Toxicol. Sci. 90 (2006) $5-22$.

[9] J. Norman, D. Politz, L. Politz, Hyperparathyroidism During Pregnancy and the Effect of Rising Calcium on Pregnancy Loss: A Call for Earlier Intervention, Clin. Endocrinol. (Oxf) 71(1) (Jul 2009) 104-109.

[10] R. Smolarczyk, J. Wójcicka-Jagodzińska, E. Romejko et al., Calcium, Phosphorus and Magnesium in Pregnant Women With Primary Hypertension, Ginekol. Pol. 68(1) (Jan. 1997) 6-10.

[11] R. Smolarczyk, J. Wójcicka-Jagodzínska, E. Romejko et al., Calcium-phosphorus-magnesium Homeostasis in Women With Threatened Preterm Delivery, Int. J. Gynaecol. Obstet. 57(1) (Apr. 1997) 43-48.

[12] B.P. Kale, M.J. Rauf, Influence of Eclipta alba on serum alanine aminotransferase and aspartate aminotransferase activity in rabbits, Int. J. of Basic \& Clin. Pharmacol. 7 (2018) 1243-1246.

[13] M.A-A. Eisa, Evaluation of total cholesterol \& glucose levels among sudanese women with preeclampsia, F EJBPS 4(1) (2017) 76-77. 